\title{
Calcium Hydroxyapatite-based Marine Origin: Novel Sunscreen Materials for Cosmeceutical Treatments
}

\author{
MOHD ZUL HELMI ROZAINI ${ }^{1 *}$, HABIBAH HAMZAH ${ }^{2}$, NOR FAZLIYANA MAHTAR MOHTAR ${ }^{2}$, \\ MOHD HASMIZAM RAZALI ${ }^{3}$, UWAISULQARNI M. OSMAN ${ }^{3}$, SABIQAH TUAN ANUAR ${ }^{4}$, \\ SITI KAMILAH CHE SOH ${ }^{4}$, SAIDATUL RADHIAH BINTI GHAZALI ${ }^{5}$, NOR HAYATI \\ IBRAHIM $^{6}$, LOW CHEN FEI ${ }^{7}$ and SHARIFAH RAHMAH ${ }^{2}$
}

${ }^{1}$ Institute of Marine and Biotechnology, Universiti Malaysia Terengganu, 21030 Kuala Nerus, Terengganu, Malaysia.

${ }^{2}$ School of Fisheries and Aquaculture Sciences, Universiti Malaysia Terengganu, 21030 Kuala Nerus, Terengganu, Malaysia.

${ }^{3}$ School of Fundamental Science, Universiti Malaysia Terengganu, 21030 Kuala Nerus, Terengganu, Malaysia.

${ }^{4}$ School of Marine Science, , Universiti Malaysia Terengganu, 21030 Kuala Nerus, Terengganu, Malaysia. ${ }^{5}$ Faculty of Chemical Engineering Technology, Tati University College, 24000, Kemaman, Terengganu, Malaysia.

${ }^{6}$ School of Technology and Food Sciences, Universiti Malaysia Terengganu, 21300 Kuala Nerus, Terengganu, Malaysia.

${ }^{7}$ Institute of Systems Biology, Universiti Kebangsaan Malaysia, 43600 Bangi, Selangor, Malaysia. ${ }^{*}$ Corresponding author: zulhelmi@umt.edu.my http://dx.doi.org/10.13005/ojc/340612

Received: October 03, 2018; Accepted: November 27, 2018)

\begin{abstract}
Fringescale sardinella or Tamban Sisik bones have been discovered can be utilized as sunscreen agent in cosmeceuticals. Its flesh is the main ingredient in keropok lekor or fish crackers in Malaysia and the bones contained very high hydroxyapatite $(\mathrm{HAp}),\left(\mathrm{Ca}_{10}\left(\mathrm{PO}_{4}\right)_{6}(\mathrm{OH})_{2}\right)$ compound which exhibit as UV light absorber. The percentage yields obtained from the hydrothermal extraction consist of $41.2 \pm 0.66 \%(\mathrm{w} / \mathrm{w})$ of HAp which was almost half of the dry weight of $100 \mathrm{~g}$ samples. The additional of manganese and ferum, initiated the novel sunscreen materials from hydroxyapatite-Fe and hydroxyapatite-Mn doped (modified bones). The unmodified HAp recorded with SPF 20 and modified HAp-Mn measured with SPF 40. Modified HAp-Fe emulsions were recorded with SPF 50 as the highest SPF value. Therefore, the bones have been characterized using Fourier Transform Infrared Radiation (FTIR) spectroscopy and X-ray diffraction (XRD). The results obtained clearly indicated that the HAp existence in waste of Fringescale sardinella bones with addition of $\mathrm{FeCl}_{2}$ which exhibits high potential as sunscreen compared to manganese and unmodified bones. Thus, the utilization of waste from the fish bones not only produce value-added products from low-cost resources, but also help in reducing pollution to the environment and preserved the global sustainability.
\end{abstract}

Keywords: Hydroxiapatite (HAp), Fishbones, sunscreen, Sun protection factor (SPF).

This is an Open Access article licensed under a Creative Commons license: Attribution 4.0 International (CC- BY). Published by Oriental Scientific Publishing Company @ 2018 


\section{INTRODUCTION}

Sunscreen agent of $\mathrm{TiO}_{2}$ and $\mathrm{ZnO}$ can potentially replace with hydroxyapatite (HAp); non toxicity calcium phosphate sources from commercial fishbones that effectively absorbs the UV region ${ }^{1,2}$. Even the $\mathrm{TiO}_{2}$ and $\mathrm{ZnO}$ were utilized as sunscreen agent but they have known their toxicity level which are harmful and affecting the users for instance lead to skin cancer ${ }^{3}$. HAp is commonly used as a filler or coating on damaged bone and implants in order to promote bone in-growth especially in orthopedic, dental, maxillofacial and biomedical applications and cosmetics ${ }^{4,5}$. Bones known as white colour, brittle properties and cheaper source that provided abundance of HAp can turn into good carriers in sunscreen ${ }^{6,7}$. It also a promising candidate for advanced sunscreens. Furthermore, its UV absorption limit can be tuned to absorb in the desired range from UVB (290-320 nm) to UVA (320-400 nm) by simply introducing a metal doping. Therefore, by doping the fish bone with proper element will initiate a huge potential of anodyne sunscreen agent as novel biomaterials and there was a study proved that iron and manganese can widen UV range absorption compared to $\mathrm{TiO}_{2}$ and $\mathrm{ZnO}^{8,9,10}$. It also help the local fishing industry especially in Terengganu (East Coast Malaysia) to manage waste disposal more wisely, improve their economic status and also preserve the environment ${ }^{11}$.

\section{Methodology \\ Extraction of Raw Materials}

The fish bones were weighed with a mass of approximately $90 \mathrm{~g}$ using analytical balance before the addition of alkaline solution. The fish bones were then divided into three beakers which contained of water, iron chloride (98\%, Merck) and manganese chloride (98\%, Merck). Each of the sample in the beaker was heated at $70^{\circ} \mathrm{C}$ for three hours and stirred. $30 \mathrm{~mL}$ of $1.0 \mathrm{M}$ of ammonium hydroxide was added into the solution of $\mathrm{FeCl}_{2}$ and $\mathrm{MnCl}_{2}$ to reduce the acidity of solution until $\mathrm{pH}$ meter indicating 8.0 $\mathrm{pH}$. The fish bones were dried again an overnight in oven and calcined at $900^{\circ} \mathrm{C}$ for three hours in a furnace. The sample was then re-weighed with analytical balance to detect the potential mass loss from the evaporation process occurred. The calcined bones were converted by grinding into powder form with high-energy ball mill for $15 \mathrm{~min}$ at $450 \mathrm{rpm}$ to yield powder.
The components and morphology of the synthesized of Fringescale sardinella bones in powder form were identified using Fourier transform infrared radiation (FTIR) and X-Ray diffraction spectrocopy (XRD).

\section{Fourier Transform Infrared Radiation (FTIR)}

FTIR was used to determine the infrared spectrum of absorption of unmodified HAp, modified $\mathrm{HAp}-\mathrm{Mn}$ and $\mathrm{HAp}-\mathrm{Fe}$. Bone powder was prepared by mixing with grinded potassium bromide $(\mathrm{KBr})$ with the 1:6 ratio. The mixture was finely pulverized and transferred into a compressor. $\mathrm{KBr}$ become plastic when subjected to pressure and form a sheet that is transparent in the infrared region. FTIR analysis of three different sunscreen agents were performed to investigate the structure of $\mathrm{HAp}$ molecules, according to the position of peak components identified in the known region. The results obtained from this analysis were expected to provide details on the different secondary structures of compound, mainly bone vibration at different peak components.

\section{X-Ray Diffraction (XRD)}

XRD was performed using Rigaku MiniFlex II diffractometer with $\mathrm{Cu} \mathrm{K \alpha}$. This test was conducted to signify the phase composition obtained in the compound of HAp, HAp-Fe and HAp-Mn. Each sample was poured and smoothly pressed in the small rectangle shape by the glass slide respectively before analyzed by the machine. Samples then were analyzed with a step of $0.02^{\circ}$, over $2 \theta$ and between the range of $10^{\circ}$ and $80^{\circ}$.

\section{Sunscreen Analysis}

The sunscreen analysis was determined to test the capability as sunscreen by In vitro method using UV Visible spectrophotometer of UV 1700 Shimadzu. An amount of $1.0 \mathrm{~g}$ of each formulated microemulsion samples was weighed and transferred into $100 \mathrm{~mL}$ of volumetric flask and then was diluted to the graduated mark with ethanol. The solution was homogenized by ultrasonicator for 5 minutes. A 5.0 $\mathrm{mL}$ of aliquot was transferred to $50 \mathrm{~mL}$ of volumetric flask and diluted to the graduated mark with ethanol. All the aliquot solution then were closed in the vials and were kept under dark condition at $25^{\circ} \mathrm{C}$. 


\section{RESULTS AND DISCUSSION}

\section{Characterization of hydroxyapatite (HAp)/ unmodified bones}

The FTIR spectrum of the HAp crystals hindmost at $600^{\circ} \mathrm{C}$ calcinations are shown at Fig. 1 (a). There is no alkyl group emerged on the spectrum thus implying the nonappearance of organic materials residual. The residual-free water was stretched and bent at broad band 3000-3800 $\mathrm{cm}^{-1}$. The bending mode of the O-H group by water absorption caused the additional band at 1600 $\mathrm{cm}^{-1}$. The $2900 \mathrm{~cm}^{-1}$ feeble band was attributed to the $\mathrm{O}-\mathrm{H}$ stretching of crystallite ${ }^{12}$. The crystal compounds were highlighted in the FTIR spectrum of Fig. 1 (b). The spectrum represented $\mathrm{PO}_{4}^{3-}$ were easily differentiate. The phosphate group bending mode (720 $\left.\mathrm{cm}^{-1}\right), \mathrm{v}_{2}$ O-P-O bending mode $\left(520-630 \mathrm{~cm}^{-1}\right)$ and $\mathrm{v}_{4}$ $\mathrm{O}-\mathrm{P}-\mathrm{O}$ bending mode bands (in the range of $430-520$ $\mathrm{cm}^{-1}$ ) are assigned and comparable to the finding by the Vallet-Regi and Gonzalez-Calbet ${ }^{13}$. Wang et al. agree with the finding of $v_{1}$ symmetric $\mathrm{P}-\mathrm{O}$ stretching mode at $900-990 \mathrm{~cm}^{-1}$ band and $\mathrm{v}_{3}$ antisymmetric $\mathrm{P}-\mathrm{O}$ at $990-1250 \mathrm{~cm}^{-1}$ band ${ }^{14}$. The pervasive $\mathrm{PO}_{4}{ }^{3-}$ bands split at $1125 \mathrm{~cm}^{-1}$ implies a high degree of crystallinity which is persistent with the XRD result.
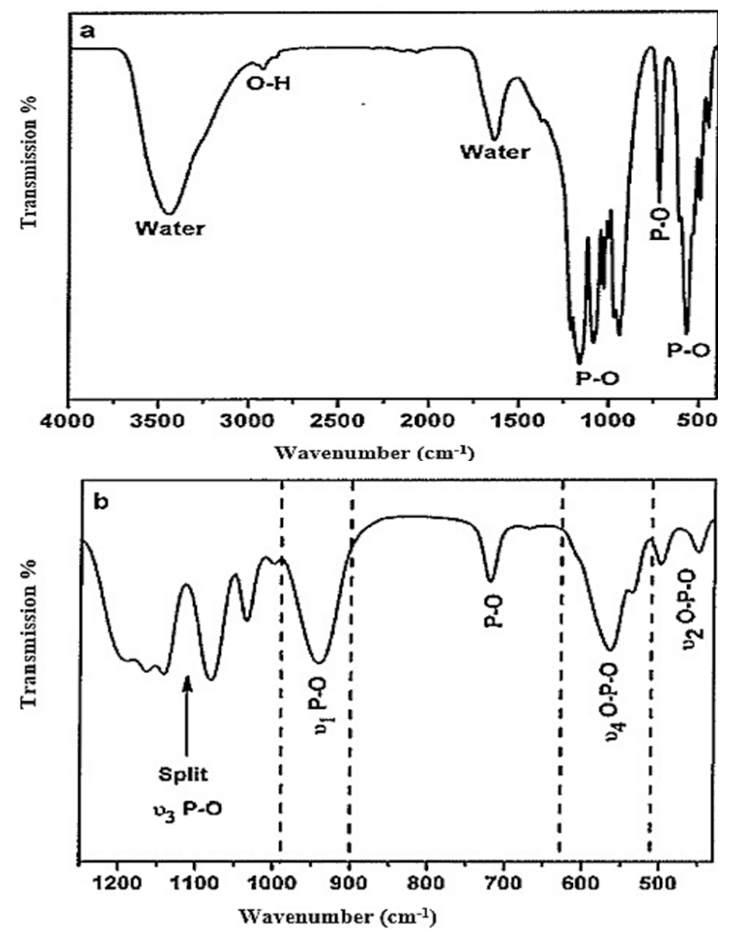

Fig. 1. FTIR spectrum of (a) unmodified HAp and (b) highlights of chemical bonding of modified HAp-Fe crystals
Figure 2 represent the typical XRD patterns of the pure $\mathrm{HAp}$ and $\mathrm{Fe}^{3+}$-doped and, $\mathrm{Mn}^{2+}$-doped HAp samples, which perfectly match with the JCPDS pattern \#74-566 for HAp. The incorporation of $\mathrm{Mn}^{2+}$ and $\mathrm{Fe}^{3+}$ into the material apparently leads to the formation of less crystalline $\mathrm{HAp}^{14}$. This effect seems natural as the dopants assumed to substitute at $\mathrm{Ca}^{2+}$ sites (ionic radius $0.99 \AA$ ) have larger charge and smaller ionic radius $\left(\mathrm{Mn}^{2+} 0.63 \AA\right.$ A $\mathrm{Fe}^{3+} 0.64 \AA$ ). Several ions, usually with an ionic radius smaller than that of $\mathrm{Ca}^{2+}$ are known to inhibit the formation of $\mathrm{HAp}^{15}$. Fig. 2(a) showed the patterns and phase composition of fish bone treated at $900^{\circ} \mathrm{C}$ with $54 \%$ of $\mathrm{HAp}, \mathrm{Ca}_{10}\left(\mathrm{PO}_{4}\right)_{6}(\mathrm{OH})_{2}$ as the main component. Fig. 2(b) showed the calcium hydrogen phosphate hydroxide, $\mathrm{Ca}_{9} \mathrm{HPO}_{4}\left(\mathrm{PO}_{4}\right)_{5} \mathrm{OH}$ as the main phase which contained about $30 \%$ but there is different phosphate-based compound, which reflected both iron and calcium - $\mathrm{Ca}_{9} \mathrm{FeH}\left(\mathrm{PO}_{4}\right)_{7} 21 \%$. A small amount of alpha hematite, $\alpha-\mathrm{Fe}_{2} \mathrm{O}_{3}$ and $\mathrm{Fe}_{2} \mathrm{O}_{3}$ also detected with $28 \%$ and $25 \%$ respectively. This indicates that iron is present with two oxidation states as $\mathrm{Fe}$ (II) and $\mathrm{Fe}$ (III) in the mixed of phosphate and hematite ${ }^{16}$.

When Mn doped with unmodified bone by mixing with $\mathrm{Mn}\left(\mathrm{NO}_{3}\right) 2 .{ }_{6} \mathrm{H}_{2} \mathrm{O}$, two different formula; Chloroapatite, $\mathrm{Ca}_{5} \mathrm{Cl}\left(\mathrm{PO}_{4}\right)_{3}$ and $\mathrm{Ca}_{10} \mathrm{Cl}_{2}\left(\mathrm{PO}_{4}\right)_{6}$ were obtained as new modified materials. Fig. 2(c) showed that, $\mathrm{Mn}$ is still presents but has been combined with phosphate producing approximately $21 \%$ of manganese chloride phosphate, $\mathrm{Mn}_{5}\left(\mathrm{PO}_{4}\right)_{3} \mathrm{Cl}$. Both modified bones resulting in the formation of $\mathrm{Fe}(\mathrm{III})$ and $\mathrm{Mn}$ (II) in the form of hematite and chloroapatite which help to prevent from UV rays ${ }^{17}$. The HAp crystals depicted much comparable diffraction profiles and XRD bulk patterns. There were no detected impurities and no particular peak of other calcium phosphate phases. The samples were well-crystallized reflected by the smooth intensity of the diffraction peaks ${ }^{18}$.

According to previous study, $\mathrm{Mn}^{2+}$-doped HAp can be obtained without producing significant alterations in the structure of $\mathrm{HAp}^{23}$. On the other hand, Le Geros reported that incorporation of reduced amounts of $\mathrm{Mn}^{2+}$ into HAp structure induces an evident reduction of the degree of crystallinity of the HAp phase ${ }^{12}$. Modified HAp-Fe exhibited high crystalline peak of reduced amounts of $\mathrm{Fe}^{3+}$ into HAp structure induces an evident reduction of the degree of crystallinity of the HAp phase. In 
this work, modified HAp-Fe doped exhibited high crystalline peak at about $33.11^{\circ}$; almost identical to the patterns that were recorded for pure and doped powders. The relatively broadening of the XRD peaks for $\mathrm{Fe}^{3+}$-doped HAp indicates that the sizes of the crystallite grains of those materials are smaller than the pure HAp and modified HAp-Mn. Through Scherrer's equation, the medium crystallite sizes were estimated. The Scherrer equation, in $\mathrm{X}$-ray diffraction and crystallography, is a formula that relates the size of sub-micrometre particles, or crystallites, in a solid to the broadening of a peak in a diffraction pattern. It is used in the determination of size of particles of crystals in the form of powder. The Scherrer equation can be written as:

$\tau=\mathrm{K} \lambda / \mathrm{B} \cos \theta$

Where $\tau$ is the mean size of the ordered (crystalline) domains, which may be smaller or equal to the grain size. $\mathrm{K}$ is a dimensionless shape factor, with a value close to unity. The shape factor has a typical value of about 0.9 , but varies with the actual shape of the crystallite. $\lambda$ is the X-ray wavelength. $\beta$ is the line broadening at half the maximum intensity, after subtracting the instrumental line broadening, in radians. This quantity is also sometimes denoted as $\Delta(2 \theta)$ and $\theta$ is the Bragg angle (in degrees).

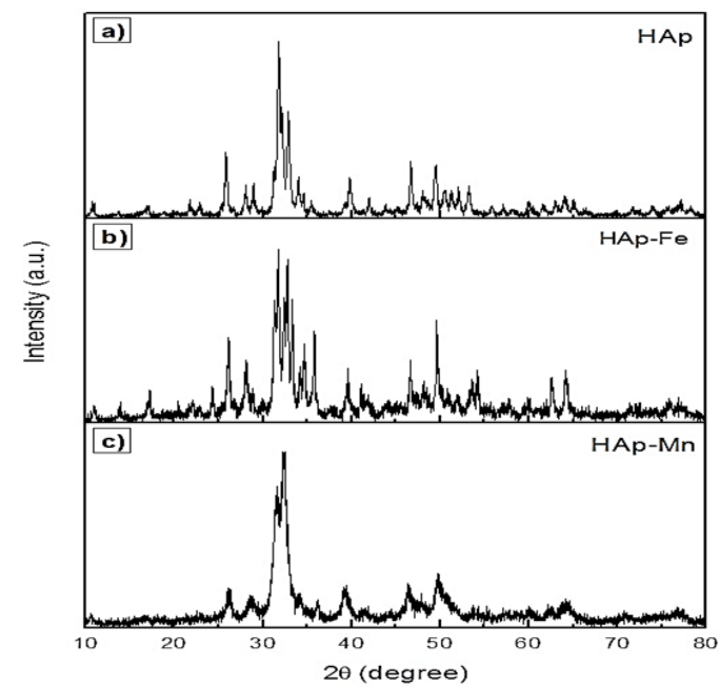

Fig. 2. X-ray diffraction pattern of (a) unmodified HAp, (b) modified HAp-Fe and (c) modified HAp-Mn doped compared to the HAp standard pattern PDF \#74-566

Note that the effect of $\mathrm{Mn}^{2+}$ on the growth of HAp particles is more remarkable than $\mathrm{Fe}^{3+}$, although the reason for these differences remain unclear at the present crystallite size as shown on Table 1.
Table 1: Maximum absorption wavelengths of the powders

\begin{tabular}{cc}
\hline Powders & Maximum Absorption $(\mathrm{nm})$ \\
\hline Pure & 207 \\
$\mathrm{Mn}^{2+}$ & $240-372$ \\
$\mathrm{Fe}^{3+}$ & $310-427$ \\
$\mathrm{TiO}_{2}$ & 316 \\
\hline
\end{tabular}

\section{Appearance, Moisture and Percentage Yield}

The physical appearance of as-synthesized and extraction sunscreen samples are compared in Table 2. Fig. 3 shows the colour appearance of the bones after the calcination process. The unmodified bones were in white ordourless apaque structures (left), the modified HAp-Fe with reddish apaque structures (middle) and modified HAp-Mn with brownish opaque structures (right). Except for colour, all samples generally had similar physical appearances. Modified HAp-Fe had the highest content of moisture $(10.3 \pm 0.13)$ and $\mathrm{pH}(7.2 \pm$ 0.32 ) compared to other samples. The phenomenon observed could be possibly explained by the highest potential of HAp yield initiated during extraction ${ }^{19}$.
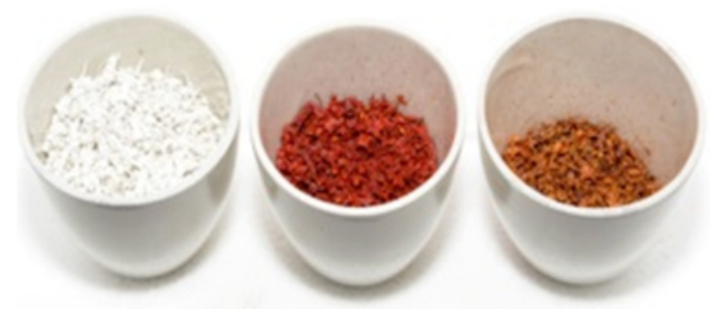

Fig. 3. The colour differences and appearance of five microemulsion formulation samples as stated in Table 3 with the combinations of different non-ionic surfactants (i.e. Span 20, Span 80, Tween 20 and Tween 80 ) with olive oil (co-surfactant) and water

The result of the unmodified HAp yield extracted was expressed as a percentage (\%) on a dry weight basis. From Table 3, the yields obtained from the extraction consist of $41.2 \pm 0.66 \%(\mathrm{w} / \mathrm{w})$ which was almost half of the dry weight of $100 \mathrm{~g}$ samples. With the moisture content of 9.6, this is more likely a maximum yield can be obtained. This also may be due to the mild conditions used in the extraction of unmodified HAp, which involved a moderate concentration of $\mathrm{NaCl}$ solution during the pre-treatment and low temperature for hot water extraction ${ }^{20,25}$. 
Table 2: Physical appearance of the investigated sunscreen agents

\begin{tabular}{|c|c|c|c|c|}
\hline \multirow[t]{2}{*}{ Sunscreen Sample } & \multicolumn{4}{|c|}{ Physical appearance } \\
\hline & Before drying & After drying & Moisture (\%) & $\mathrm{pH}(1 \%)$ \\
\hline & White o & White opaque powder & $05^{\mathrm{b}}$ & 6.3 \\
\hline d HAp & White strong odorous & White opaque powder & 8.12 & $0.12^{\mathrm{a}}$ \\
\hline Modified Hap-Fe & White odorous powder & Reddish odorless powder & 10.3 & $7.2 \pm 0.32^{b}$ \\
\hline Modified Hap-Mn & White odorous powder & Brownish odorless powder & $9.16 \pm 0.03^{b}$ & $7.1 \pm 0.22^{b}$ \\
\hline Zinc oxide, (ZnO) & Yellow odorless powder & Yellowish odorless powder & $9.19 \pm 0.1^{b}$ & $7.5 \pm 0.23^{a}$ \\
\hline
\end{tabular}

Values are the mean \pm standard deviation of triplicate samples

${ }^{a-b}$ Means with the same superscripst within a row are not significantly different $(P<0.05)$

Table 3: Physical parameters of sunscreen samples

\begin{tabular}{lccc}
\hline & Yield (\%) & Melting Point $\left({ }^{\circ} \mathrm{C}\right)$ & Viscosity $(\mathrm{Pa})$ \\
\hline Commercial Hydroxyapatite & NA & 16.2 & 9.1 \\
Unmodified HAp & $41.2 \pm 0.66^{\mathrm{b}}$ & $18.6 \pm 0.42^{\mathrm{a}}$ & $18.8 \pm 0.55^{\mathrm{a}}$ \\
Modified HAp-Fe & NA & $26.9 \pm 0.65^{\mathrm{a}}$ & $20.8 \pm 0.65^{\mathrm{b}}$ \\
Modified HAp-Mn & NA & $29.1 \pm 0.26^{\mathrm{c}}$ & $19.0 \pm 0.26^{\mathrm{b}}$ \\
\hline
\end{tabular}

Values are the mean \pm standard deviation of triplicate samples

${ }^{a-b}$ Means with the same superscripst within a row are not significantly different $(P<0.05)$

Yield (weight of hydroxyapatite per $100 \mathrm{~g}$ of dry Unmodified Tamban bones)

NA: Not Applicable

\section{Sunscreen Analysis}

Cosmeticians are more interested in finding In vitro methods to evaluate the photoprotection abilities of different sunscreen compounds ${ }^{9}$. There are two common In vitromethods: (a) UV radiation is transmitted through sunscreen product films in quartz plates or biomembranes or (b) the measurement of absorption characteristics using spectrophotometric analysis ${ }^{9,24}$.

Additionally, the in vitro method can be substituted by the following equation with the aid of UV spectrophotometry?:

$$
\mathrm{SPFx}_{\text {spectrophotometric }}=\mathrm{CF} \times \sum_{290}^{320} \mathrm{EE}(\tau) \times \mathrm{I}(\tau) \times \mathrm{Abs}(\tau) \text { (2) }
$$

Where: $E E(I)$ - erythemal effect spectrum; I (I) - solar intensity spectrum; Abs (I)- absorbance of sunscreen product; $\mathrm{CF}$ - correction factor $(=10)$. The values of $E E x I$ are constants.

The emulsion formulation was analysed to obtain the sun protection factor (SPF) value using Mansur equation in order to measure the tendency as protector from direct UV-rays ${ }^{21}$. This is because the SPF is represent the effectiveness of a sunscreen formulation ${ }^{22}$. The calculated SPF values on emulsion, emulsion HAp, emulsion HAp-Fe and emulsion HAp-Mn was recorded in Table 4.

Table 4: SPF value of the sunscreen formulation

\begin{tabular}{cccc}
\hline Formulation & Active Ingredients & Amount (\%) & Sunscreen Protection Factor (SPF) \\
\hline N1 & 0 & 0 & $9.630 \pm 1.5$ \\
N2 & unmodified HAp & 3.0 & $23.533 \pm 1.3$ \\
N4 & modified HAp-Fe & 3.0 & $48.940 \pm 1.2$ \\
N3 & modified HAp-Mn & 3.0 & $40.469 \pm 1.6$ \\
\hline
\end{tabular}

All values are presented as Mean $\pm S D(n=3)$

The result has shown that the SPF measurement for emulsion HAp-Fe was $48.94 \pm$ 0.55 which achieved nearly SPF 50 and this led to the emulsion performed as a good sunscreen absorber than other emulsions. The emulsion HAp-Mn was shown that the SPF value was 240.56 \pm 0.49 . The SPF measurement of emulsion HAp-Mn was lower than emulsion HAp-Fe because of the dependence on the type of metal attachment with the fish bones ${ }^{11}$. The iron was claimed as having more potential in prevention of UV rays rather than $\mathrm{Mn}$. The SPF measurement of emulsion HAp was 
$9.11 \pm 0.60$.. This is because the fish bones had not altered and that is the reason the measurement value of emulsion HAp was lower at $23.49 \pm 0.65$. It means that the material of the standard emulsion itself has possibility to shield dangerous UV rays. Therefore, it was clearly seen that the emulsion HAp-Fe was shown as a great sunscreen due to the high absorption value among the emulsion ${ }^{16}$.

\section{CONCLUSION}

This research has proved the concept of innovating waste to wealth. Local resources have been explored thoroughly in order to create something very useful to community along with the smartest way on utilize the waste. Tamban (Fringescale sardinella) bones collected from factory waste are currently emerged as a potential and promising application for the sunscreen formulation in cosmetics. This study investigated the role of HAp derived from Tamban bone and the modification of unmodified HAp with the ferum and manganese doped in microemulsion structure, which was used as a reaction medium for the synthesis of novel sunscreen agents. The percentage yields obtained from the extraction consist of $41.2 \pm 0.66 \%(\mathrm{w} / \mathrm{w})$ which was almost half of the dry weight of $100 \mathrm{~g}$ bone samples. Pure HAp (unmodified Tamban bone) recorded with SPF 20 and modified HAp-Mn doped measured with SPF 40. Modified HAp-Fe microemulsions were recorded with SPF 50 and the highest SPF value. This is really a good indicator where we can synthesize more ferrous metal doping into HAp to increase their absorbance as well as SPF values.

\section{ACKNOWLEDGEMENT}

This research was supported Ministry of Higher Education Malaysia (MOHE) by the Fundamental Research Grant Scheme initiative (via FRGS 59423/SG01/2016) and Niche Research Grant Scheme (NRGS) [NRGS53131/STWN/2015]. We thank our colleagues Mr Nasrul Yahya from Technology Park Malaysia, Raub who provided insight and expertise that greatly assisted the research, with all of the interpretations of this paper and also to all volunteers who made this investigation possible.

\section{REFERENCES}

1. Burnett, M. E. \& Wang, S. Q. Current sunscreen controversies: A Critical Review. Photodermatology, Photoimmunology \& Photomedicine., 2011, 27 (2), 58-67.

2. Boutinguiza, M., Pou, J., Comesaña, R., Lusquiños, F., Carlos, A. De, \& León, B. Materials Science \& Engineering C., 2012, 32 (3). 478-486.

3. Araujo, T. S., Souza, S O., Sousa, E. M. B., Araújo, M. S. Production and Thermal Stability of Pure and $\mathrm{Cr}^{3+}$-doped Hydroxyapatite., 2010, 249, 1-7.

4. Piccrillo, C., Rocha, C., Tobaldi, D. M., Pullar, R. C., Labrincha, J. A., Ferreira, M. O., Castro, P. M. L., Pintado, M. M. E. J. Mater. Chem. B., 2014, 2, 5999-6009.

5. Ferraro, V., Carvlho, A. P., Santos, M. M., Castro, P. M. L., Pintado, M. E. Mat Sci Eng C-Mater., 2013, 33, 3111-3120.
6. Wang, C., Chang, T., Shi, L., Yang, H., Cui, M., Tambalu, L. Seafood Processing ByProducts: Collagen and Gelatin. Kim, S.-K. (ed.). Trend and Applications. New York: Springer Science., 2013.

7. Springsteen, A., Yurek, R., Frazier, M., Carr, K F. In vitro Measurement of Sun Protection Factor of Sunscreens by Diffuse Transmittance1, Analytica Chimica Acta., 1999, 380, 155-164.

8. Miyaji F, Kono Y, \& Suyama Y. Materials Research Buletin., 2005, 40, 209-220.

9. Mansur, J.S.; Breder, M.N.R.; Mansur, M.C.A; Azulay, R.D An. Bras. Dermatol., 2010, 61, 121-124.

10. Rao S. Journal of Indian Chemical Society., 1976, 9, 344-347.

11. Rozaini M.Z.H, Hamzah, H. Journal of Sustainability Science and Management., 
2017, 3, 75-84.

12. LeGeros R. Z., Taheri M. M, Quirologico G. B., \& Le Geros J. P. Proceeding of $2^{\text {nd }}$ International Phosphorus Conference, Boston, United States., 1989, 89-103.

13. Harry R. G., Harry's cosmeticology 13th edition Leonard Hill Books(London)., 2003.

14. Brezova, V. \& Stasko A. Journal of Catalysis., 1994, 147, 156-162.

15. Pinnell S.R., David Fairhurst, Robert Gillies \& Nikiforos Kollias. Dermatology Surgical., 2008, 26(4), 309-314.

16. Dindar, B., \& Icli S. J. Photochem. Photobiol. A. Chem., 2001, 140, 263-268.

17. Rozaini M.Z.H., Ali R.C, \& Rose L.C. Int. Journal of Tech., 2012, 3(2), 103-109.
18. Nishikawa, H. Material Letters., 2001, 50(5), 364-370.

19. Flor J. \& Davolos M. R. Quimica. Nova., 2007, 32, 5-10.

20. Harry R. G., Harry's cosmeticology 13th edition Leonard Hill Books (London)., 2003.

21. Panda A, Patel P. N. \& Panda A. Indian Journal of Chem., 1989, 29, 62-70.

22. Razali, M. H., \& Yusoff, M. Materials Letters., 2018, 221, 168-171.

23. Panda A, Patel P N, Panda A. Indian J Chem., 1989, 29, 62-70.

24. Pissavini M, Ferrero L. Cosmets. Toiletries, Oak Park., 2003, 118, 63-72.

25. Rozaini M.Z.H. Orient. J. Chem., 2012, 28(2), 803-808. 